Original

\title{
Evaluation of depression, anxiety and stress levels in patients with oral lichen planus
}

\author{
Barbara Manczyk ${ }^{1,2)}$, Joanna Gołda2), Agnieszka Biniak2), Katarzyna Reszelewska2), \\ Bernadetta Mazur2), Karolina Zając2), Paulina Bińczak2), \\ Maria Chomyszyn-Gajewska ${ }^{3)}$, and Zuzanna Oruba ${ }^{3)}$ \\ 1)Central Ambulatory, University Dental Clinic, Kraków, Poland \\ 2)Student Research Group, Chair of Periodontology and Oral Medicine, Faculty of Medicine, \\ Jagiellonian University Medical College, Kraków, Poland \\ 3)Chair of Periodontology and Oral Medicine, Faculty of Medicine, Jagiellonian University Medical College, \\ Kraków, Poland
}

(Received March 31, 2018; Accepted September 25, 2018)

\begin{abstract}
The pathogenesis of oral lichen planus (OLP) remains to be fully elucidated; however, certain psychoneurological factors may influence the onset and exacerbation of OLP. The aim of the present study is to evaluate the intensity of negative emotions in patients with OLP. A cross-sectional, questionnairebased study was performed. The sample consisted of 52 subjects, comprising 26 patients with OLP (OLP group) and 26 controls (CTRL group). The Depression Anxiety Stress Scale 21 (DASS-21) was used for psychometric evaluation. The patients were also asked about their attitude toward the disease, treatment, and interference of the disease on daily life. The mean level of depression was $12.54 \pm 6.6$ in the OLP group and 7.69 \pm 5.22 in the CTRL group $(P=$ 0.006). The mean level of anxiety was $11.15 \pm 7.95$ in the OLP group and $6.62 \pm 4.86$ in the CTRL group ( $P$ $=0.018)$. The mean stress levels were $8.69 \pm 7.06$ and $3.85 \pm 3.18$ in the OLP and CTRL groups, respectively $(P=0.003)$. Severe and very severe scores of depression and very severe scores of anxiety and stress were
\end{abstract}

Correspondence to Dr. Zuzanna Oruba, Faculty of Medicine, Jagiellonian University Medical College, Montelupich 4, Kraków 31-155, Poland

Fax: +48-12-424-54-320 E-mail: zuzanna.oruba@uj.edu.pl

J-STAGE Advance Publication: June 10, 2019

doi.org/10.2334/josnusd.18-0113

DN/JST.JSTAGE/josnusd/18-0113 present in the OLP group, whereas these emotions were normal in the majority of controls. Depression, stress, and anxiety may be involved in the pathogenesis and course of OLP.

Keywords: oral lichen planus; depression; anxiety; stress; DASS-21.

\section{Introduction}

Lichen planus (LP) is a chronic inflammatory mucocutaneous disease, which affects $1-2 \%$ of the general population (1). Lesions most frequently occur on cutaneous and oral surfaces $(40 \%)$ or are solitary on either the skin or mucosa alone (35\% and $25 \%$, respectively) (2). Mucosal manifestations most commonly affect the oral mucosa, although other mucous membranes, including the genitalia, esophagus, and conjunctiva, may also be involved. Cutaneous presentation, apart from the flexor regions of the legs and arms, may also affect skin appendages, including the scalp and nails (3).

Within the oral cavity, there are six variants of oral lichen planus (OLP) lesions: papular, plaque-like, erosive, bullous, atrophic, and reticular (4). Among these, the reticular type is the most prevalent and is usually asymptomatic, whereas the erosive type, although less frequent, is of a greater clinical significance due to its symptoms, which range from slight discomfort and burning sensation to episodes of severe pain that inter- 
feres with speaking, eating, and swallowing (5). OLP is estimated to be the most frequent noninfectious oral mucosal disease among patients referred to oral medicine and oral pathology clinics (1).

The precise etiology of OLP remains to be fully elucidated, and there are several hypotheses regarding the pathogenesis and possible etiological and risk factors of the disease. There is evidence that a cell-mediated autoimmune reaction in which $\mathrm{CD} 4^{+}$and $\mathrm{CD} 8^{+} \mathrm{T}$ lymphocytes induce the apoptosis of epithelial basal cells may be involved (5). It has also been suggested that oxidative stress may be of importance in the pathogenesis of OLP (6). Studies have shown a correlation between decreased serum levels of an important antioxidant, uric acid, with exacerbation of the LP state compared with healthy controls and patients with LP in the remission phase (7). In addition, psychological disorders including stress, anxiety, and depression have frequently been reported as possible factors associated with the onset and/or exacerbation of OLP $(3,8,9)$. Stress is the most commonly patient-reported trigger of OLP flare-ups $(10,11)$. In addition, patient-perceived symptom improvement resulting from avoidance of this trigger is similar to that obtained following active pharmacological treatment (11). To support this hypothesis, higher levels of cortisol in serum, saliva, and urine have been detected in patients with OLP compared with those in healthy subjects (12). With respect to these data, pharmacological and/ or psychotherapeutic management of stress may serve as a beneficial adjunct strategy in the treatment of OLP. Therefore, psychological evaluation may be a valuable step in the holistic diagnosis of patients with OLP.

The Depression Anxiety Stress Scale (DASS) comprises a questionnaire that was developed in 1995 by Lovibond to provide a self-reported measure of anxiety, depression, and stress (13). The original DASS consisted of 42 items; however, a shorter version (DASS-21) was developed to reduce administration time and has been widely used in clinical samples to screen for symptoms at different levels of depression, anxiety, and stress (14). Each questionnaire is divided into three subscales measuring the three dimensions of negative emotional states, depression (DASS-D), anxiety (DASS-A), and stress (DASS-S). Depression refers to low levels of positive affect (dysphoria, hopelessness, lack of energy, and anhedonia). Anxiety refers to irritability, agitation, difficulty relaxing, and impatience. Stress is characterized by persistent tension, a low threshold of becoming upset or frustrated, and a tendency to overreact to stressful events (14).

The primary aim of the present study is to evaluate the intensity of negative emotions (anxiety, stress, and depression) in patients with OLP. The secondary aim is to identify a possible correlation between the extent of depression, anxiety, and stress and the patients' attitude toward the disease, treatment, and disease interference on daily life.

\section{Study design \\ Materials and Methods}

A cross-sectional questionnaire-based study was conducted. The study protocol was approved by the Bioethical Committee at the Jagiellonian University in December 2016 (approval no. 122/6120/352/2016).

\section{Study population}

The present study included two groups of patients: the study group (OLP group) and the control group (CTRL group). The OLP group consisted of patients referred for a control appointment to the Outpatient Clinic of Periodontology and Clinical Oral Pathology, University Dental Clinic in Kraków (Poland) with a diagnosis of OLP based upon histopathological examination. The CTRL group consisted of patients without the disease referred to the University Dental Clinic for a consultation not related to oral mucosal lesions. The exclusion criteria for both groups were as follows: 1) age $<18$ years; 2) congenital or acquired immune system deficiencies; 3 ) systemic drug therapies affecting the immune system; 4) history of mental illness and ongoing or past pharmacological psychiatric therapy; and 5) pregnancy and lactation. Patients who were willing to participate in the study and able to read and understand unassisted were included. All patients provided their informed consent on receiving a full explanation of the purpose of the study, and there were no financial implications for the patients. A total of 26 patients were enrolled into each group.

\section{Questionnaire}

The DASS-21 was applied to obtain data. The questionnaire consists of 21 symptoms divided into three subscales of seven items to evaluate, respectively, depression, anxiety, and stress. The patients were asked to rate the extent to which they had experienced each symptom over the previous week on a four-point scale ranging between 0 ("did not apply to me at all") and 3 ("applied to me very much or most of the time"). Subsequently, the total number of points for each subscale was calculated and multiplied by two. Based on the score obtained, the extent of a given negative emotion was classified as normal, mild, moderate, severe, or extremely severe (13).

The second part of the questionnaire comprised 
Table 1 Demographic characteristics of patients

\begin{tabular}{lcc}
\hline Characteristic & OLP (study group) & CTRL (control group) \\
\hline Group size & 26 & 26 \\
Age (mean \pm SD) & $63.12 \pm 13.01$ & $51.46 \pm 9.09$ \\
Gender: & & \\
male & $8(30.8 \%)$ & $12(46.2 \%)$ \\
female & $18(69.2 \%)$ & $14(53.8 \%)$ \\
Education: & & \\
primary/vocational & $5(19.2 \%)$ & $2(7.7 \%)$ \\
secondary & $14(53.8 \%)$ & $9(34.6 \%)$ \\
tertiary & $7(26.9 \%)$ & $15(57.7 \%)$ \\
Marital status: & & \\
single & $2(7.7 \%)$ & $1(3.8 \%)$ \\
married & $17(65.4 \%)$ & $21(80.8 \%)$ \\
divorced & $2(7.7 \%)$ & $2(7.7 \%)$ \\
widowed & $5(19.2 \%)$ & $2(7.7 \%)$ \\
\hline
\end{tabular}

OLP: oral lichen planus.

questions concerning demographics, systemic health, and socioeconomic status. The questions asked for the patients' perception of the efficacy of treatment, quality of the cooperation with the oral pathologist, adherence to given recommendations, and interference of the disease on daily life. The answers to these questions were graded from "definitely negative" to "definitely positive." These questions were asked to verify whether the perceived arduousness of the disease correlated with the extent of depression, anxiety, and stress.

\section{Statistical analysis}

The data were pooled in Microsoft Excel and analyzed using Statistica 10 and IBM SPSS (the software supplied by the Jagiellonian Univeristy, Kraków, Poland). The groups were compared in terms of the mean levels of negative emotions by the means of Student's $t$-test for independent samples upon verification of a normal distribution with the Shapiro-Wilk test. Subsequently, in order to compare the distribution of scores of negative emotions between the study and control groups, a chi-square test of independence was applied. This tool enables determination of the significance of the relationship between two nominal (categorical) variables, i.e., the presence of the disease (OLP vs. CTRL) and the score of a specific emotion (normal, mild, moderate, severe, or very severe) (7). The same test was used to evaluate the association between the intensity of negative emotions and patient-perceived aspects of disease and treatment. For these comparisons, in which significant results for the chi-square test were obtained, additional post hoc analysis was performed by computing adjusted residuals in cross tabulations. The level of significance was established as $P<0.05$. The Bonferroni correction was applied for residual analysis.

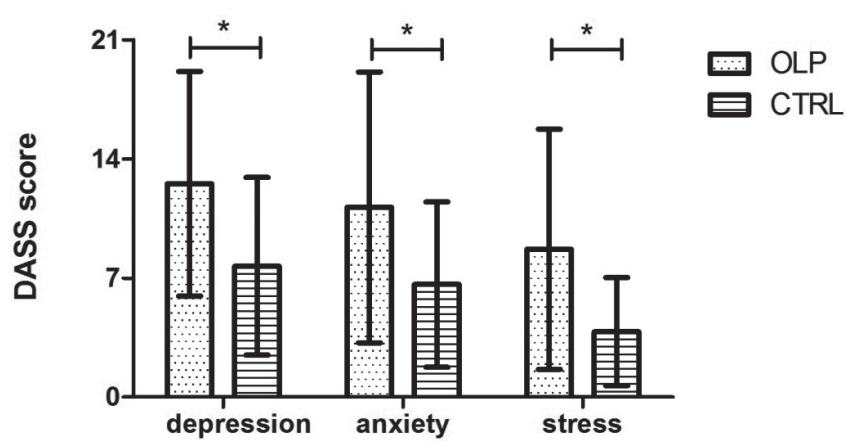

Fig. 1 Mean levels of depression, anxiety, and stress in OLP and CTRL groups. ${ }^{*} P<0.05$. OLP: oral lichen planus; CTRL: control; DASS: Depression Anxiety Stress Scale.

\section{Results}

The present study included 52 patients, with 26 individuals in each group. The demographic characteristics of the study population are presented in Table 1 .

The patients with OLP exhibited significantly higher mean levels of depression, anxiety, and stress compared with the control subjects (Fig. 1). The mean level of depression was $12.54 \pm 6.6$ in the OLP group and 7.69 \pm 5.22 in the CTRL group $(P=0.006)$. The mean level of anxiety was $11.15 \pm 7.95$ in the OLP group and $6.62 \pm$ 4.86 in the CTRL group $(P=0.018)$. The mean levels of stress were $8.69 \pm 7.06$ and $3.85 \pm 3.18$ in the OLP and CTRL groups, respectively $(P=0.003)$ (Fig. 1).

The distributions of the scores of depression, anxiety, and stress in the OLP and CTRL groups are presented in Tables 2-4.

Severe $(11.5 \%)$ and very severe $(3.8 \%)$ levels of depression were reported in the OLP study group only. The majority of control subjects (69.2\%) exhibited a normal level of depression. Significant differences between the OLP and CTRL groups were observed $(P=$ $0.018)$. The residual statistical analysis revealed that the groups differed significantly in terms of the distribution of the normal score of depression, which was lower in the OLP group (Table 2).

Although $19.2 \%$ of patients with OLP exhibited very severe levels of anxiety, compared with none in the CTRL group, and the number of patients with anxiety in the range of normal was almost twice as high in the CTRL $(61.5 \%)$ than the OLP group (34.6\%), there were no significant differences between the two groups in the distribution of the scores $(P=0.090)$ (Table 3$)$.

Regarding the magnitude of stress, $100 \%$ of the participants in the CTRL group scored normal grade of stress. By contrast, patients in the OLP group reported 
Table 2 Comparison of depression scores (DASS-21 scale) between the study and control groups

\begin{tabular}{lcccc}
\hline Depression & $\begin{array}{c}\text { OLP } \\
\text { (study group) }\end{array}$ & $\begin{array}{c}\text { CTRL } \\
\text { (control group) }\end{array}$ & Total & $\begin{array}{c}P \text {-value for post hoc } \\
\text { comparison }\end{array}$ \\
\hline Normal & $7(26.9 \%)$ & $18(69.2 \%)$ & 25 & $P=0.002$ \\
Mild & $7(26.9 \%)$ & $2(7.7 \%)$ & 9 & $P=0.067$ \\
Moderate & $8(30.8 \%)$ & $6(23.1 \%)$ & 14 & $P=0.529$ \\
Severe & $3(11.5 \%)$ & - & 3 & $P=0.075$ \\
Very severe & $1(3.8 \%)$ & - & 1 & $P=0.313$ \\
Total & 26 & 26 & 52 & \\
\hline$\chi^{2}=11.904 ; \mathrm{df}=4 ; P=0.018 ;$ significant. OLP: oral lichen planus; DASS-21: Depression Anxiety Stress Scale 21.
\end{tabular}

Table 3 Comparison of anxiety scores (DASS-21 scale) between the study and control groups

\begin{tabular}{lccr}
\hline Anxiety & $\begin{array}{c}\text { OLP } \\
\text { (study group) }\end{array}$ & $\begin{array}{c}\text { CTRL } \\
\text { (control group) }\end{array}$ & Total \\
\hline Normal & $9(34.6 \%)$ & $16(61.5 \%)$ & 25 \\
Mild & $1(3.8 \%)$ & $2(7.7 \%)$ & 3 \\
Moderate & $7(26.9 \%)$ & $6(23.1 \%)$ & 13 \\
Severe & $4(15.4 \%)$ & $2(7.7 \%)$ & 6 \\
Very severe & $5(19.2 \%)$ & - & 5 \\
Total & 26 & 26 & 52 \\
\hline
\end{tabular}

$\chi^{2}=8.037 ; \mathrm{df}=4 ; P=0.090 ;$ not significant. OLP: oral lichen planus.

Table 5 Intensity of depression depending on gender in patients with oral lichen planus

\begin{tabular}{lccc}
\hline Depression & Male & Female & Total \\
\hline Normal & $2(7.69 \%)$ & $5(19.23 \%)$ & 7 \\
Mild & $2(7.69 \%)$ & $5(19.23 \%)$ & 7 \\
Moderate & $3(11.54 \%)$ & $5(19.23 \%)$ & 8 \\
Severe & $1(3.85 \%)$ & $2(7.69 \%)$ & 3 \\
Very severe & - & $1(3.85 \%)$ & 1 \\
Total & 8 & 18 & 26 \\
\hline$\chi^{2}=0.656 ; \mathrm{df}=4 ; P=0.957 ;$ not significant.
\end{tabular}

Table 7 Intensity of stress depending on gender in patients with oral lichen planus

\begin{tabular}{lccc}
\hline Stress & Male & Female & Total \\
\hline Normal & $6(23.08 \%)$ & $15(57.69 \%)$ & 21 \\
Mild & $2(7.69 \%)$ & $1(3.85 \%)$ & 3 \\
Moderate & - & $1(3.85 \%)$ & 1 \\
Severe & - & $1(3.85 \%)$ & 1 \\
Very severe & - & - & - \\
Total & 8 & 18 & 26 \\
\hline$\chi^{2}=2.751 ; \mathrm{df}=3 ; P=0.432 ;$ not significant. & &
\end{tabular}

mild (11.5\%), moderate (3.8\%), and severe (3.8\%) levels of stress. Although the scores higher than normal scores were reported in the OLP group only, this was not statistically significant $(P=0.14)$.

The possible correlations between gender and the intensity of the negative emotions among patients with OLP were also investigated. The mean levels of depres-
Table 4 Comparison of stress scores (DASS-21 scale) between the study and control groups

\begin{tabular}{lccc}
\hline Stress & $\begin{array}{c}\text { OLP } \\
\text { (study group) }\end{array}$ & $\begin{array}{c}\text { CTRL } \\
\text { (control group) }\end{array}$ & Total \\
\hline Normal & $21(80.8 \%)$ & $26(100.0 \%)$ & 47 \\
Mild & $3(11.5 \%)$ & - & 3 \\
Moderate & $1(3.8 \%)$ & - & 1 \\
Severe & $1(3.8 \%)$ & - & 1 \\
Very severe & - & - & 0 \\
Total & 26 & 26 & 52 \\
\hline$\chi^{2}=5.53191 ; \mathrm{df}=3 ; P=0.14 ;$ not significant. OLP: oral lichen planus;
\end{tabular}

DASS-21 scale.

Table 6 Intensity of anxiety depending on gender in patients with oral lichen planus

\begin{tabular}{lccc}
\hline Anxiety & Male & Female & Total \\
\hline Normal & $4(15.38 \%)$ & $5(19.23 \%)$ & 9 \\
Mild & - & $1(3.85 \%)$ & 1 \\
Moderate & $2(7.69 \%)$ & $5(19.23 \%)$ & 7 \\
Severe & $2(7.69 \%)$ & $2(7.69 \%)$ & 4 \\
Very severe & - & $5(19.23 \%)$ & 5 \\
Total & 8 & 18 & 26 \\
\hline$\chi^{2}=4.167 ; \mathrm{df}=4 ; P=0.384 ;$ not significant.
\end{tabular}

sion, anxiety, and stress in female patients with OLP were $12.78 \pm 6.7,12.56 \pm 8.19$, and $9.22 \pm 7.37$, respectively, whereas the respective levels in male patients were $12 \pm$ $6.32,8 \pm 6.32$, and $7.5 \pm 6.14$. Although the tendency for higher anxiety and stress values was observed in females, this was not statistically significant. Similarly, in terms of the distributions of the scores of depression, anxiety, and stress, higher scores were observed more frequently in females; however, again, no significant differences were observed (Tables 5-7).

Subsequently, patients with OLP were asked about their attitude toward the disease and aspects of treatment (Table 8). The majority of patients rated their cooperation with the main doctor as good (92.3\%). Patient-perceived efficacy of treatment was most frequently assessed as good or rather good (34.5\% and $38.5 \%$, respectively). When asked about adherence to given recommendations related to the treatment of OLP (including topical appli- 
Table 8 Evaluation of patients' attitudes toward the disease and treatment

\begin{tabular}{|c|c|c|c|c|}
\hline Question & Response of OLP group & $\begin{array}{l}\text { Correlation with } \\
\text { depression scores }\end{array}$ & $\begin{array}{l}\text { Correlation with } \\
\text { anxiety scores }\end{array}$ & $\begin{array}{l}\text { Correlation with } \\
\text { stress scores }\end{array}$ \\
\hline $\begin{array}{l}\text { How do you rate the quality of the } \\
\text { cooperation with the main doctor? }\end{array}$ & $\begin{array}{l}\text { Good: } 24(92.3 \%) \\
\text { Rather good: } 2(7.7 \%) \\
\text { Hard to determine: } 0 \\
\text { Rather bad: } 0 \\
\text { Bad: } 0\end{array}$ & $\begin{array}{l}\mathrm{x}^{2}=4,288 \\
\mathrm{df}=4 \\
P=0.368 \\
\mathrm{~ns}\end{array}$ & $\begin{array}{l}\mathrm{x}^{2}=1.14 \\
\mathrm{df}=4 \\
P=0.842 \\
\mathrm{~ns}\end{array}$ & $\begin{array}{l}\mathrm{x}^{2}=0.516 \\
\mathrm{df}=3 \\
P=0.915 \\
\text { ns }\end{array}$ \\
\hline $\begin{array}{l}\text { How do you evaluate the effectiveness } \\
\text { of treatment? }\end{array}$ & $\begin{array}{l}\text { Good: } 9(34.5 \%) \\
\text { Rather good: } 10(38.5 \%) \\
\text { Hard to determine: } 6(23.1 \%) \\
\text { Rather bad: } 1(3.9 \%) \\
\text { Bad: } 0\end{array}$ & $\begin{array}{l}\mathrm{x}^{2}=10.455 \\
\mathrm{df}=12 \\
P=0.576 \\
\mathrm{~ns}\end{array}$ & $\begin{array}{l}\mathrm{x}^{2}=8.382 \\
\mathrm{df}=12 \\
P=0.755 \\
\mathrm{~ns}\end{array}$ & $\begin{array}{l}\mathrm{x}^{2}=9.217 \\
\mathrm{df}=9 \\
P=0.417 \\
\text { ns }\end{array}$ \\
\hline $\begin{array}{l}\text { Do you adhere to given recommenda- } \\
\text { tions? }\end{array}$ & $\begin{array}{l}\text { Yes: } 1(3.9 \%) \\
\text { Partially: } 23(88.5 \%) \\
\text { No: } 2(7.6 \%)\end{array}$ & $\begin{array}{l}\mathrm{x}^{2}=6.729 \\
\mathrm{df}=8 \\
P=0.566 \\
\mathrm{~ns}\end{array}$ & $\begin{array}{l}\mathrm{x}^{2}=4.881 \\
\mathrm{df}=8 \\
P=0.77 \\
\mathrm{~ns}\end{array}$ & $\begin{array}{l}\mathrm{x}^{2}=0.807 \\
\mathrm{df}=6 \\
P=0.992 \\
\text { ns }\end{array}$ \\
\hline $\begin{array}{l}\text { How much does the disease interfere } \\
\text { with your daily life? }\end{array}$ & $\begin{array}{l}\text { Significantly: } 5(19.2 \%) \\
\text { Moderately: } 12(46.2 \%) \\
\text { Not at all: } 9(34.6 \%)\end{array}$ & $\begin{array}{l}\mathrm{x}^{2}=6.63 \\
\mathrm{df}=8 \\
P=0.577 \\
\mathrm{~ns}\end{array}$ & $\begin{array}{l}\mathrm{x}^{2}=9.808 \\
\mathrm{df}=8 \\
P=0.279 \\
\text { ns }\end{array}$ & $\begin{array}{l}\mathrm{x}^{2}=9.575 \\
\mathrm{df}=6 \\
P=0.144 \\
\mathrm{~ns}\end{array}$ \\
\hline
\end{tabular}

OLP: oral lichen planus; ns: not significant.

cation of corticosteroids and/or retinoids, complying with oral hygiene regimen, avoiding alcohol and tobacco, and avoiding spicy, acidic, and hot food and beverages), only one patient $(3.9 \%)$ reported they had fully adhered to all advice, whereas the majority only partially adhered. Almost half of the respondents admitted that the disease interfered moderately with their daily lives.

No statistically significant associations between the patient-perceived aspects of disease and treatment mentioned above and the ranges of either depression, anxiety, or stress were detected (Table 8).

\section{Discussion}

The aetiopathogenesis of OLP is complex and remains to be fully elucidated. Numerous genetic, environmental, and lifestyle factors appear to be of importance (3). The role of emotional disturbances in the pathogenesis and course of the disease is also frequently postulated (8).

The present study aims to evaluate the extent of depression, anxiety, and stress in patients with OLP by means of DASS-21. The highest scores of depression, i.e., severe and very severe, were reported only among the OLP patient group (11.5\% and 3.8\%, respectively). By contrast, the majority of control subjects $(69.2 \%)$ exhibited normal levels of depression. In addition, the number of patients with anxiety in the range of normal was almost twice as high in the CTRL group (61.5\%) than in the OLP group (34.6\%), whereas a very severe level of anxiety was noted in the OLP group only. The level of stress was in the range of normal for $100 \%$ of control subjects, whereas mild $(11.5 \%)$, moderate
(3.8\%), and severe (3.8\%) levels of stress were reported in the OLP group. For depression statistically significant differences between the OLP and CTRL groups were observed for both mean values and the distribution of scores. For anxiety and stress, the mean values in the two groups differed significantly; however, the difference in the distribution of scores was not statistically significant, although a tendency for higher scores in the OLP group was noted. Additionally, higher mean values of anxiety and stress and a more frequent prevalence of higher scores for negative emotions were reported in females with OLP than males. However, due to the lack of statistical significance, inequality in the number of females and males, and low overall number of participants, this tendency should be interpreted with caution. This may be a future direction of investigations in understanding the role of negative emotions in the aetiopathogenesis of OLP.

The psychometric evaluation of patients with OLP using DASS has been conducted in two previous studies. In a study by Gupta et al., in which the shortened version of the questionnaire was also applied, similar outcomes to those described in the present study were reported. The depression and stress scores were significantly different between the control and OLP groups. Only patients in the OLP group exhibited severe levels of depression (12.8\%) and stress $(15.4 \%)$, whereas the levels of these two variables in the control group were rated as normal, mild, and moderate. The anxiety scores were comparable in both groups and did not differ significantly (7). Kalkur et al., in turn, applied DASS-42 and concluded that patients 
with OLP exhibited a higher frequency of psychiatric comorbidities, including depression, anxiety, and stress, compared with the control group. However, there were no significant differences in the mean values between the OLP and control groups for these three variables (15).

Psychometric evaluation of the patients with OLP was also performed by Chaudhary (16). Two questionnaires were used: the General Health Questionnaire-version 28 (GHQ-28) by Goldberg and Hillier for the assessment of psychosocial stressors and the Hospital Anxiety and Depression Scale by Zigmond and Snaith, which was applied to determine the levels of depression and anxiety. Chaudhary divided patients into three groups: OLP, negative control (patients without mucosal diseases), and positive control (patients suffering from diseases in which the etiology is influenced by psychosocial factors, including burning mouth syndrome and atypical facial pain). Significantly higher scores of depression, anxiety, and stress were observed in patients with OLP and positive controls when compared with negative controls. However, there were no statistically significant differences between the OLP group and positive control. The author concluded that psychological stressors are important causative factors in OLP and other autoimmune reactions (16).

Rojo-Moreno et al. conducted a study involving 100 patients with OLP and 50 controls (17). They used the following tools for the purpose of the psychometric evaluation: Spielberger State-Trait Anxiety Inventory, Cattell Personality Questionnaire 16PF, Hassanyeh Rating of Anxiety-Depression-Vulnerability, Beck Depression Inventory, Raskin Depression Screen, and Covi Anxiety Screen. All applied scales indicated higher scores of anxiety and depression in patients with OLP than controls. Furthermore, it was found that patients with OLP differed significantly from controls in terms of the expression of personality features, including emotional sensitivity, tendency to experience feelings of guilt, inflexibility, and vulnerability to psychic disorders. In turn, when the patients with non-erosive and erosive LP were compared, the latter exhibited a higher intensity of depression. It was concluded that despite higher anxiety scores in patients with OLP, it was not possible to determine whether the observed psychological alterations constitute a direct cause of OLP or are, on the contrary, a consequence of OLP and its lesions (17).

The greater intensities of anxiety, depression, and stress have also confirmed in other studies using questionnaire-based approaches (18-23), and stress remains the most commonly patient-reported trigger of OLP lesions $(10,11)$. In addition to increased vulnerability to stress, patients with LP are often concerned about the possibility of malignancy, which may contribute to the further potentiation of emotional tension (24).

Patients with OLP also report poorer sleep quality, measured using the Pittsburgh Sleep Quality Index, when compared with healthy subjects $(21,23)$. The association between depression, anxiety, daytime sleepiness, and sleep quality in patients with OLP was significant in these two studies.

The levels of cortisol in saliva in patients with OLP have also been a focus of research. Cortisol, a stress hormone, is released upon activation of the hypothalamicpituitary-adrenal axis and is considered an indicator of psychological stress. It has been shown that patients with OLP exhibit significantly higher saliva cortisol levels than non-OLP controls $(12,18,20,23)$. Additionally, higher cortisol levels are correlated with increased intensity of anxiety $(18,20)$. Karthikeyan et al. compared the level of cortisol in the saliva, serum, and urine of patients with OLP, recurrent aphthous stomatitis (RAS), and healthy controls. The levels of this hormone were significantly elevated in all three body fluids in both OLP and RAS when compared with those in controls (12). Lopez-Jornet et al. showed that in addition to cortisol, salivary $\operatorname{IgA}$ was elevated in patients with OLP, indicating depressed immune function (23).

Although the results of the studies cited above and those presented in the present study indicate a greater intensity of negative emotions in patients with OLP, they do not fully clarify whether OLP results from increased anxiety and depression or actually causes increased emotional tension by inducing fear of cancer. Studies reporting on stress as a main trigger of OLP flares appear to indirectly confirm that emotional factors may be involved in the aetiopathogenesis of the disease $(10,11)$. However, it has also been mentioned that it is the anxiety of cancerization in the course of OLP that is responsible for the poor psychological well-being in these patients $(17,24)$. This unexplained phenomenon highlights the importance of future research on OLP.

The secondary aim of the present study is to identify a possible correlation between the extent of depression, anxiety, and stress and the patients' attitude toward aspects related to disease and treatment. The following aspects were considered: quality of the cooperation with the oral pathologist, effectiveness of treatment, adherence to recommendations, and interference of the disease on daily life. No statistically significant correlations were detected. However, Lundquist et al. found a positive correlation between high scores of stress and patient-perceived interference of the disease with daily 
life, work, and social/spare time in patients with erosive oral and genital LP (19).

In conclusion, the present study confirmed that OLP is associated with elevated levels of depression, anxiety, and stress. These results suggest the possible role of negative emotions and psychological disturbances in the aetiopathogenesis and course of OLP. Patients with OLP may require a multidisciplinary approach, including psychological evaluation and support, in order to optimize diagnosis and treatment.

However, the results of the present study should be considered in the light of several limitations. Firstly, a cross-sectional study design enabled evaluation only at a single timepoint during the course of the disease and thus does not allow precise cause-effect assessment. Secondly, the patients with OLP were not differentiated based on the type of disease (reticular vs. erosive LP). Such differentiation of patients may be important due to the severity of symptoms in erosive LP which may potentiate emotional tension in patients. Therefore, further well-designed clinical studies on larger populations are essential to elucidate the association between psychological disturbances and ORL, which may be bidirectional.

\section{Conflict of interest}

The authors have no conflict of interest to declare.

\section{References}

1. Sugerman PB, Savage NW (2002) Oral lichen planus: causes, diagnosis and management. Aust Dent J 47, 290-297.

2. De Rossi SS, Ciarrocca K (2014) Oral lichen planus and lichenoid mucositis. Dent Clin North Am 58, 299-313.

3. Nogueira PA, Carneiro S, Ramos-e-Silva M (2015) Oral lichen planus: an update on its pathogenesis. Int J Dermatol 54, 1005-1010.

4. Di Stasio D, Guida A, Salerno C, Contaldo M, Esposito V, Laino L et al. (2014) Oral lichen planus: a narrative review. Front Biosci 6, 370-376.

5. Payeras MR, Cherubini K, Figueiredo MA, Salum FG (2013) Oral lichen planus: focus on etiopathogenesis. Arch Oral Biol 58, 1057-1069.

6. Rekha VR, Sunil S, Rathy R (2017) Evaluation of oxidative stress markers in oral lichen planus. J Oral Maxillofac Pathol 21, 387-393.

7. Gupta A, Mohan RP, Gupta S, Malik SS, Goel S, Kamarthi N (2017) Roles of serum uric acid, prolactin levels, and psychosocial factors in oral lichen planus. J Oral Sci 59, 139-146.

8. Mohamadi Hasel K, Besharat MA, Abdolhoseini A, Alaei Nasab S, Niknam S (2013) Relationships of personality factors to perceived stress, depression, and oral lichen planus severity. Int J Behav Med 20, 286-292.
9. Gavic L, Cigic L, Biocina Lukenda D, Gruden V, Gruden Pokupec JS (2014) The role of anxiety, depression, and psychological stress on the clinical status of recurrent aphthous stomatitis and oral lichen planus. J Oral Pathol Med 43, 410-417.

10. Eisen D (2002) The clinical features, malignant potential, and systemic associations of oral lichen planus: a study of 723 patients. J Am Acad Dermatol 46, 207-214.

11. Chen HX, Blasiak R, Kim E, Padilla R, Culton DA (2017) Triggers of oral lichen planus flares and the potential role of trigger avoidance in disease management. Oral Surg Oral Med Oral Pathol Oral Radiol 124, 248-252.

12. Karthikeyan P, Aswath N (2016) Stress as an etiologic co-factor in recurrent aphthous ulcers and oral lichen planus. J Oral Sci 58, 237-240.

13. Lovibond PF, Lovibond SH (1995) The structure of negative emotional states: comparison of the Depression Anxiety Stress Scales (DASS) with the Beck Depression and Anxiety Inventories. Behav Res Ther 33, 335-343.

14. Crawford JR, Henry JD (2003)The Depression Anxiety Stress Scales (DASS): normative data and latent structure in a large non-clinical sample. Br J Clin Psychol 42, 111-131.

15. Kalkur C, Sattur AP, Guttal KS (2015) Role of depression, anxiety and stress in patients with oral lichen planus: a pilot study. Indian J Dermatol 60, 445-449.

16. Chaudhary S (2004) Psychosocial stressors in oral lichen planus. Aust Dent J 49, 192-195.

17. Rojo-Moreno JL, Bagan JV, Rojo-Moreno J, Donat JS, Milian MA, Jimenez Y (1998) Psychologic factors and oral lichen planus. A psychometric evaluation of 100 cases. Oral Surg Oral Med Oral Pathol Oral Radiol Endod 86, 687-691.

18. Koray M, Dulger O, Ak G, Horasanli S, Ucok A, Tanyeri H et al. (2003) The evaluation of anxiety and salivary cortisol levels in patients with oral lichen planus. Oral Dis 9, 298-301.

19. Lundqvist EN, Wahlin YB, Bergdahl M, Bergdahl J (2006) Psychological health in patients with genital and oral erosive lichen planus. J Eur Acad Dermatol Venereol 20, 661-666.

20. Nadendla LK, Meduri V, Paramkusam G, Pachava KR (2014) Association of salivary cortisol and anxiety levels in lichen planus patients. J Clin Diagn Res 8, 1-3.

21. Adamo D, Ruoppo E, Leuci S, Aria M, Amato M, Mignogna MD (2015) Sleep disturbances, anxiety and depression in patients with oral lichen planus: a case-control study. J Eur Acad Dermatol Venereol 29, 291-297.

22. Alves MG, do Carmo Carvalho BF, Balducci I, Cabral LA, Nicodemo D, Almeida JD (2015) Emotional assessment of patients with oral lichen planus. Int J Dermatol 54, 29-32.

23. Lopez-Jornet P, Cayuela CA, Tvarijonaviciute A, Parra-Perez F, Escribano D, Ceron J (2016) Oral lichen planus: salival biomarkers cortisol, immunoglobulin A, adiponectin. J Oral Pathol Med 45, 211-217.

24. Burkhart NW, Burkes EJ, Burker EJ (1997) Meeting the educational needs of patients with oral lichen planus. Gen Dent 45, 126-132. 